\title{
Probe of Multielectron Dynamics in Xenon by Caustics in High-Order Harmonic Generation
}

\author{
D. Faccialà, ${ }^{1,2}$ S. Pabst,${ }^{3,4}$ B. D. Bruner, ${ }^{5}$ A. G. Ciriolo, ${ }^{1,2}$ S. De Silvestri, ${ }^{1,2}$ M. Devetta, ${ }^{2}$ M. Negro, ${ }^{2}$ \\ H. Soifer, ${ }^{5}$ S. Stagira, ${ }^{1,2}$ N. Dudovich, ${ }^{5}$ and C. Vozzi ${ }^{2, *}$ \\ ${ }^{1}$ Dipartimento di Fisica, Politecnico di Milano, 20133 Milan, Italy \\ ${ }^{2}$ Istituto di Fotonica e Nanotecnologie-CNR, 20133 Milan, Italy \\ ${ }^{3}$ Center for Free-Electron Laser Science DESY, 22607 Hamburg, Germany \\ ${ }^{4}$ ITAMP Harvard-Smithsonian Center for Astrophysics, 02138 Cambridge, Massachusetts, USA \\ ${ }^{5}$ Department of Physics of Complex Systems, Weizmann Institute of Science, 7610001 Rehovot, Israel
}

(Received 4 March 2016; published 26 August 2016)

\begin{abstract}
We investigated the giant resonance in xenon by high-order harmonic generation spectroscopy driven by a two-color field. The addition of a nonperturbative second harmonic component parallel to the driving field breaks the symmetry between neighboring subcycles resulting in the appearance of spectral caustics at two distinct cutoff energies. By controlling the phase delay between the two color components it is possible to tailor the harmonic emission in order to amplify and isolate the spectral feature of interest. In this Letter we demonstrate how this control scheme can be used to investigate the role of electron correlations that give birth to the giant resonance in xenon. The collective excitations of the giant dipole resonance in xenon combined with the spectral manipulation associated with the two-color driving field allow us to see features that are normally not accessible and to obtain a good agreement between the experimental results and the theoretical predictions.
\end{abstract}

DOI: 10.1103/PhysRevLett.117.093902

High-order harmonic generation (HHG) has proved to be a valuable spectroscopic tool for probing the electron structure [1,2] and dynamics [3-5] of atoms and molecules. In this process an electron wave packet can be detached from the atom by tunnel ionization at each half-cycle of the driving laser field. This electron wave packet is then accelerated by the external field and can recollide with the parent ion, releasing its excess energy through the emission of an extreme ultraviolet (XUV) burst $[6,7]$. Each burst has the duration of a few hundred attoseconds, which makes HHG the cornerstone for the generation of the shortest events ever created. The photon energy $\hbar \omega$ is linked to the time of birth of the electron in the continuum $t_{i}$ and to its recombination time $t_{r}$, defining a quantum trajectory [8]. Since these processes occur on a time scale shorter than the laser electric field cycle, and owing to the mapping between photon energies and electron trajectories, attosecond time resolution can be inferred through the analysis of HHG spectra.

One of the main challenges in attosecond science is the ability to manipulate the optical properties of the HHG spectrum, such as the spectral shape, polarization, and phase. Such manipulations are the basic building blocks in a broad range of applications where the attosecond pulse

Published by the American Physical Society under the terms of the Creative Commons Attribution 3.0 License. Further distribution of this work must maintain attribution to the author(s) and the published article's title, journal citation, and DOI. serves as a pump or a probe of a fast evolving process. In this Letter we show how an accurate manipulation of the HHG spectrum amplifies and isolates the spectral features associated with the attosecond dynamics under study. When the HHG is generated by a two-color field, an accurate control over its spectral shape can be achieved. Such control leads to the appearance of a spectral caustic [9], allowing a tunable enhancement of a narrow spectral band. We engineered the HHG spectrum and tuned the caustic such that it overlaps with the spectral feature associated with the giant resonance in xenon (Xe), demonstrating how the application of such control serves as a valuable tool in HHG spectroscopy.

In a single color driving field, two trajectories contribute to the HHG signal for each photon energy, namely, the short and long trajectories [8]. The spectrum extends up to the cutoff energy $\hbar \omega_{\text {cutoff }}=1.32 I_{p}+3.17 U_{p}$, where $I_{p}$ is the ionization potential of the target and $U_{p}$ is the ponderomotive energy of the free electron in the field. Approaching the cutoff, these two trajectories merge and only one trajectory is physically allowed $[8,10,11]$. When HHG is driven by the combination of the fundamental field with its second harmonic in parallel polarization, the symmetry between neighboring subcycles is broken and each trajectory is split in two distinct ones that recollide with the ion from opposite sides. This eventually leads to the presence of two distinct cutoff energies [12-14]. Figure 1(a) shows the two semiclassical cutoffs as a function of the phase delay $\varphi$ between the two colors. 

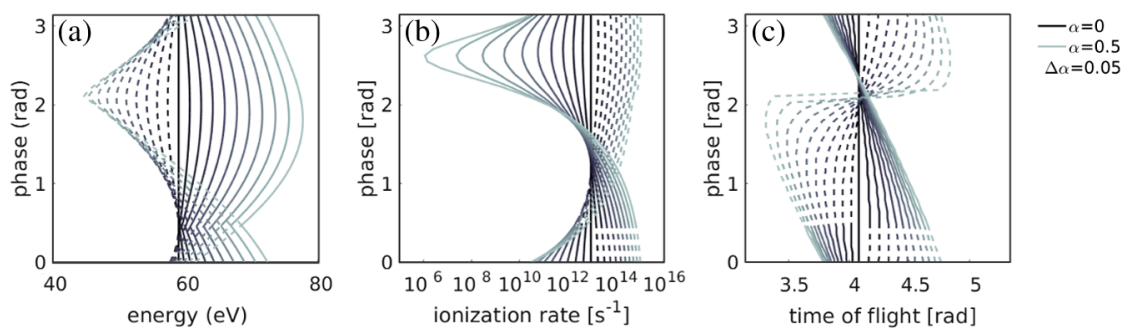

FIG. 1. Photon energies (a), ionization rates (b), and excursion times (c) associated to the cutoff trajectories as a function of the phase difference between the two fields for different values of $\alpha$ ranging from 0 to 0.5 with step $\Delta \alpha=0.05$. The dashed line represents the lower branch and the solid line represents the upper branch. The two cutoff energies are calculated with the semiclassical three-step model; the ionization rates of the two cutoff trajectories are calculated with the Ammosov-Delone-Krainov model [15]; the excursion times of the two cutoff trajectories are calculated with the semiclassical three-step model and they are normalized to the period of the fundamental field. The intensity of the fundamental field is equal to $I=7 \times 10^{13} \mathrm{~W} / \mathrm{cm}^{2}$.

The cutoff energy is shown for different values of the ratio between the two field components, $\alpha$, ranging from 0 to 0.5 with step $\Delta \alpha=0.05$. We can identify a lower branch (lower cutoff, dashed line) and an upper branch (upper cutoff, solid line). Approaching the cutoff the spectral density diverges to infinity [9], leading to the appearance of a spectral caustic and allowing an enhancement of a narrow bandwidth in this spectral region. When more than two trajectories coalesce in the same spectral region this produces a dramatic enhancement as predicted by catastrophe theory. The appearance of the caustic identifies the spectral regime where the stationary solution is singular, providing a clear fingerprint of the well-known strong-field analysis.

By changing $\varphi$ and $\alpha$, the appearance of the caustic can then be tuned to overlap with the spectral region of interest. However, in the experiment the visibility of the caustic also depends on the ionization probability and electron wave packet spreading of the associated cutoff trajectory. In Figs. 1(b) and 1(c) the ionization probabilities and the excursion times of the recolliding electron for the two cutoff trajectories are reported. It is possible to observe that the lower branch has, for most of the values of $\varphi$, a higher ionization probability than the upper branch. In the remaining regions, namely, for $\varphi$ approximately between 0.4 and $1.2 \mathrm{rad}$, the excursion time is higher, which leads to a higher spreading of the electronic wave function and a lower recombination probability [12]. For these reasons, the upper branch has never been clearly observed in the experiments. However, if the caustic overlaps a shape resonance of the target sample, the spectral focusing associated with the caustic combined with the enhanced cross section could efficiently counteract the reduction of the intensity, making the full spectral features available for being probed.

As a benchmark case, we applied two-color HHG for probing the giant resonance in xenon, a very broad enhancement in the harmonic spectrum around $100 \mathrm{eV}$ that has been predicted by Frolov et al. [16] and experimentally observed by Shiner et al. [17]. This enhancement was attributed to the multielectron inelastic scattering that takes place during the recombination of the electron with the parent ion. The electron ionized from the $5 p$ valence shell can recombine to the ground state by two possible channels. Either it can directly recombine with the $5 p$ hole or it can exchange energy by inelastic scattering with one of the underlying $4 d$ electrons, which is promoted to the $5 p$ valence shell, leaving a hole in the $4 d$ state. Eventually, the electron recombines with the $4 d$ hole. This recombination channel can be accessed if the kinetic energy of the recolliding electron exceeds the energy difference between the two orbitals involved. This interpretation has been confirmed by time-dependent configuration-interaction singles (TDCIS) calculations taking into account the many-body interaction between different inner-shell orbitals $[18,19]$. TDCIS is a many-body theory that can capture multiorbital effects in the attosecond [20,21] and strongfield regimes [21-23], and has been successfully applied to capture the behavior of the collective dipole resonance in xenon in the presence of an intense XUV [24] and IR pulses [25].

The quantitative comparison between experimental results and single atom predictions is usually very difficult. One of the main problems arises from the fact that the intensity of the HHG spectrum can be shaped by phasematching effects [26-30]. HHG is in fact the coherent buildup of the single atom emissions of a macroscopic sample excited by a laser pulse [31]. For low pressure target media, phase matching is essentially determined by the balancing between two terms: the geometrical phase shift (Gouy phase) and the dipole phase, which depends linearly on the driving intensity $I$, through a constant that takes different values according to the trajectory considered $[32,33]$. This leads to a different conversion efficiency for the two families of trajectories in the plateau. Two-color HHG is particularly favorable since at the caustic two trajectories coalesce in a single one [34], providing a clear and controllable spectral feature that can be tuned around a wide spectral range.

The experimental setup is described in detail in [35]. The HHG driving pulse is a carrier envelope phase stabilized 
pulse at $\lambda_{1}=1550 \mathrm{~nm}$ with a time duration of $25 \mathrm{fs}$ [36]. The second field component is the second harmonic of the fundamental pulse, generated inside a $\beta$-barium borate crystal. We used a calcite plate to correct the group delay between the two pulses and a birefringent retardation plate for rotating the polarization of the fundamental field, thus switching to parallel polarization configuration. A pair of fused silica wedges was used to fine-tune the phase delay between the two laser fields. The harmonics were generated by focusing the two-color driving field into a pulsed gas jet. In order to reduce phase-matching effects the backing pressure of the gas behind the valve was kept below 1 bar. In addition, the gas nozzle was positioned away from the laser focus and its diameter was much smaller than the beam Rayleigh range. The harmonic field was acquired by an XUV flat-field spectrometer [37] followed by a microchannel plate and a phosphor screen coupled to a CCD camera.

Figure 2(a) shows a sequence of harmonic spectra acquired in xenon as a function of the two-color phase delay $\varphi$. Both caustics corresponding to the lower and the upper branches are clearly visible. The strong enhancement of the lower caustic at $\varphi \approx 2.1 \mathrm{rad}$ around $45 \mathrm{eV}$ is due to the coalescence of four electron trajectories. For this unique point all the four first time derivatives of the quasiclassical action vanish, forming a swallowtail caustic [9]. The unique behavior of the two caustics as a function of $\varphi$ and $\alpha$ allows the estimation of $\alpha$ and of the driving peak intensity of the fundamental color $I$ in the interaction region with a high level of accuracy from the comparison with the semiclassical calculations in Fig. 1. The best fit corresponds to
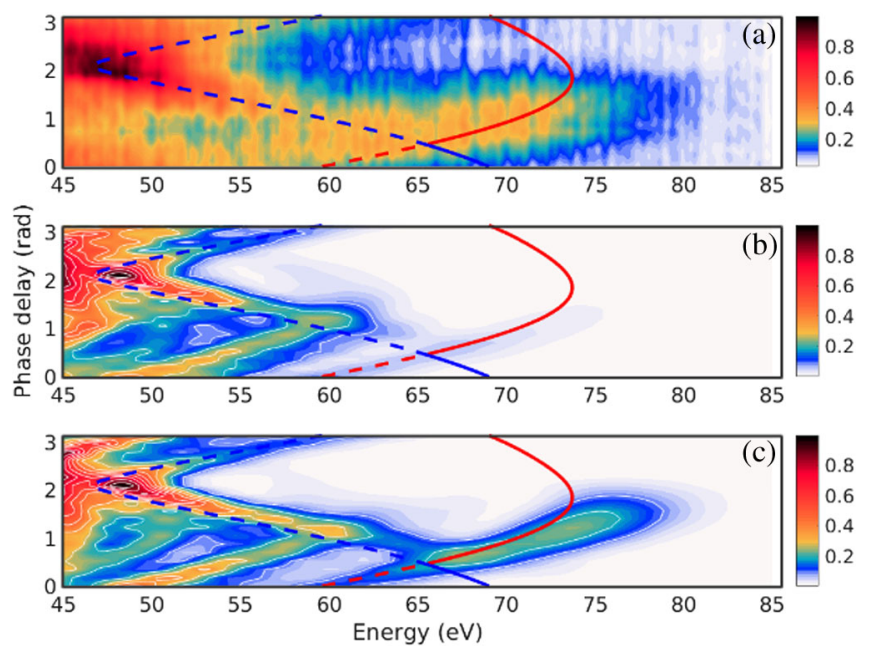

FIG. 2. HHG spectra as a function of the phase delay between the two colors-linear scale color map. (a) Experiment (b) TDCIS simulations with only the $5 p_{z}$ orbital being active. (c) TDCIS simulations with all $5 p, 4 d$, and $5 s$ orbitals being active. The solid and the dashed curves represent the two cutoffs for the upper and lower branches, respectively, associated with the semiclassical calculations.
$I=7 \times 10^{13} \mathrm{~W} / \mathrm{cm}^{2}$ and $\alpha=0.404$. The calculated cutoffs according to the semiclassical model are reported on top of the color map.

Figures 2(b) and 2(c) show the calculated HHG spectrum based on the TDCIS method with (b) only the $5 p_{z}$ orbital active and (c) with all orbitals in the $4 d, 5 s$, and $5 p$ shells active. HHG is calculated using the time-dependent dipole moment $\langle z\rangle(t)$. Restricting the manifold of active occupied orbitals to $5 p_{z}$ mimics the single-active electron (SAE) picture where the returning electron can only recombine with the originally occupied $5 p_{z}$ orbital. Figure 2(b) shows that the upper branch is basically not visible in the HHG spectrum in the SAE picture due to the spreading of the electron wave packet and especially due to the reduced ionization probability of the corresponding electron trajectories. The experimental results in Fig. 2(a) show that major parts of the upper branch are visible, which is inconsistent with the SAE picture. Including the $4 d, 5 s$, and $5 p$ shells and their interaction in the calculations leads to a very good agreement between theory and experiment [see Figs. 2(a) and 2(c)]. The experimental evidence of the existence of the upper branch has already been acquired in neon [38]. However, the conversion efficiency of the upper branch is very low when collective resonance effects are not present due to the suppression already discussed in the singleactive electron picture. Our results demonstrate that the enhanced recombination amplitude, due to the coupling between the $4 d$ and $5 p$ shells, counteracts the reduction in the tunnel ionization probability, and enables the measurement of spectral features that are normally inaccessible in HHG. This allows us to precisely determine the position of the two cutoffs and to compare them with the theoretical calculation for each value of the two-color phase.

In our measurements it is possible to clearly distinguish the two caustics of the upper and lower branch and tune their spectral position with a high level of accuracy. In these conditions it is also possible to directly compare the experimental results with the TDCIS calculations. In Fig. 3 the maximum HHG intensity with respect to the phase delay $\varphi$ from the experimental data is shown as a function of the photon energy as a solid line. The predicted HHG intensities from TDCIS calculations with (dashed line) and without (dotted line) the contribution of the $4 d$ and $5 s$ orbitals are also shown. The calculations are in very good agreement with the experimental data only if the multielectron effects are taken into account. A good quantitative agreement is achieved in the spectral region where only one trajectory is selected (50-60 and $67-80 \mathrm{eV})$. The aforementioned results show that the analysis of the caustics in nonperturbative two-color HHG provides a unique insight into the underlying mechanism. The position of the upper branch can be predicted via a simple single electron strong-field analysis. The estimation of its strength requires a multielectron analysis. This response reveals that the interaction in the vicinity of the 


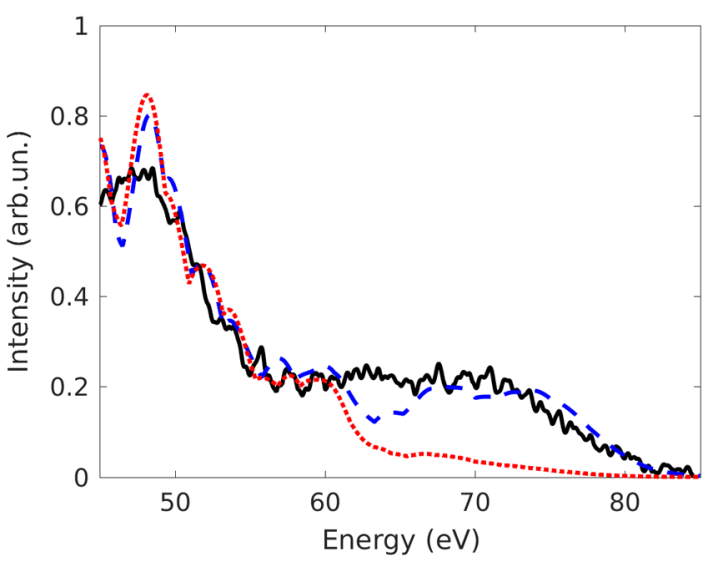

FIG. 3. Maximum value of HHG intensity (linear scale) with respect to the phase delay as a function of the photon energy. (Solid line) Experiment. (Dotted line) TDCIS simulations considering only $5 p$ orbitals. (Dashed line) TDCIS simulations including all $5 p, 4 d$, and $5 s$ orbitals.

resonance can be described via the single electron strongfield picture, while the multielectron interaction dictates its prefactor only.

From the TDCIS calculations it is also possible to extract the time structure of the HHG field as shown in Fig. 4. The Gabor transform of the calculated HHG spectra taking into account all contributing orbitals is shown in panel (a) for
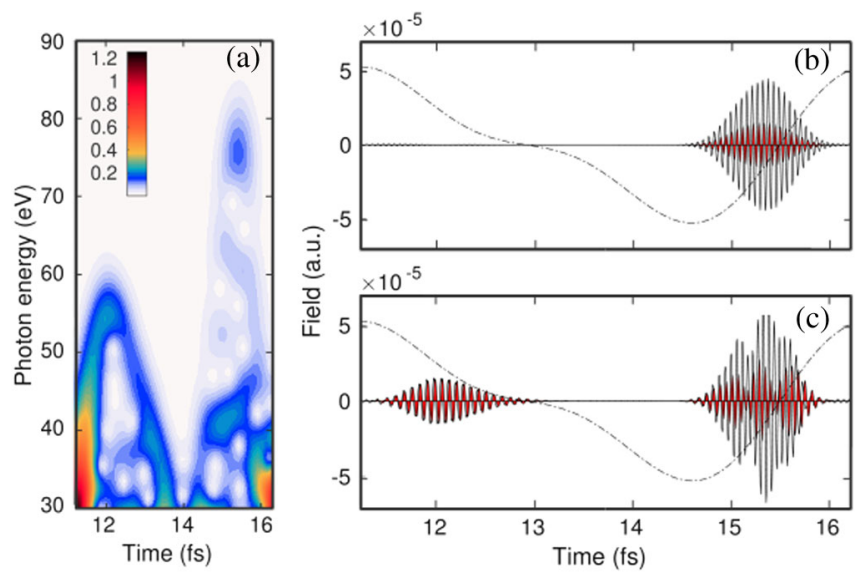

FIG. 4. (a) Gabor (time-frequency) analysis of the HHG spectra calculated with the TDCIS approach including all $5 p, 4 d$, and $5 s$ orbitals (Gaussian time gate $\sigma=86$ as). (b) Temporal structure of the HHG field calculated with the TDCIS approach including all $5 p, 4 d$, and $5 s$ orbitals (solid line) and considering only $5 p$ orbitals (lighter area) in the energy region corresponding to $75 \pm 5 \mathrm{eV}$. The driving electric field is shown as the dash-dotted curve (value divided by $10^{3}$ ). (c) Temporal structure of the HHG field calculated with the TDCIS approach including all $5 p, 4 d$, and $5 s$ orbitals (solid line) and considering only $5 p$ orbitals (lighter area) in the energy region corresponding to $75 \pm 15 \mathrm{eV}$. The driving electric field is shown as a dash-dotted curve (value divided by $10^{3}$. All calculations refer to one period of the driving field and $\varphi=0.5 \pi \mathrm{rad}$. the particular case of $\varphi=0.5 \pi \mathrm{rad}$ and for one period of the driving field. The two caustics at 55 and $75 \mathrm{eV}$ correspond to the points where long and short trajectories converge. Figure 4(b) shows the temporal structure of the harmonic electric field in the spectral region of the giant resonance $(70-80 \mathrm{eV})$ with and without the contribution of the $4 d$ and $5 s$ orbitals. The spectral filtering isolates only the contribution of the upper caustic, which is enhanced by the multielectron effect. If a broader spectral region between 60 and $90 \mathrm{eV}$ is selected, as in Fig. 4(c), two attosecond bursts appear associated to the trajectories giving rise to the lower and upper branch around 12 and 15 fs, respectively. As is clear from the results in Fig. 4(c), only the attosecond burst associated to the upper branch is enhanced by the shape resonance.

In conclusion, we studied HHG in xenon driven by a nonperturbative two-color field. With this technique, we have shown how one can reveal and enhance a number of features in the HHG spectrum of Xe that are often hidden in other types of HHG spectroscopy. The relatively strong second harmonic field breaks the symmetry between neighboring half-cycles leading to two cutoffs that are energetically well separated and enhanced by spectral focusing. The upper branch, which is normally not visible in the experiments due to the strongly reduced tunneling and recombination rates, is clearly visible in xenon because of the collective excitation involving the $4 d, 5 s$, and $5 p$ shells. A direct comparison between the experimental and theoretical results confirms that the upper branch is only visible due to the collective dipole excitation in xenon. The analysis of the spectrum at the caustic offers a reliable probe for comparing the experiment with single atom predictions. Importantly, it reveals that the excitation of the resonance dynamics can be described via a single electron strong-field picture, while the multielectron interaction dictates its strength. We expect that these results can be extended beyond investigations of the structure of the giant resonance in xenon [39], and can pave the way toward more general HHG measurements of electron correlations and structural features such as shape resonances in many other atomic and molecular systems. For instance, caustic trajectories can offer a reliable probe for investigating and disentangling structural and multielectron dynamic features in aligned molecular samples.

The research leading to these results has received funding from the European Research Council Starting Research Grant UDYNI (Grant No. 307964) and from the Italian Ministry of Research and Education (ELI Grant No. ESFRI Roadmap). S. P. is funded by the Alexander von Humboldt Foundation and by the NSF through a grant to ITAMP. N. D. acknowledges the Minerva Foundation, the Israeli Science Foundation, the Israeli Centers of Research Excellence program, the Crown Photonics Center, and the European Research Council Starting Research Grant MIDAS. 
*caterina.vozzi@ifn.cnr.it

[1] J. Itatani, J. Levesque, D. Zeidler, H. Niikura, H. Pépin, J. C. Kieffer, P. B. Corkum, and D. M. Villeneuve, Nature (London) 432, 867 (2004).

[2] C. Vozzi, M. Negro, F. Calegari, G. Sansone, M. Nisoli, S. D. Silvestri, and S. Stagira, Nat. Phys. 7, 822 (2011).

[3] W. Li, X. Zhou, R. Lock, S. Patchkovskii, A. Stolow, H. C. Kapteyn, and M. M. Murnane, Science 322, 1207 (2008).

[4] O. Smirnova, Y. Mairesse, S. Patchkovskii, N. Dudovich, D. Villeneuve, P. Corkum, and M. Y. Ivanov, Nature (London) 460, 972 (2009).

[5] D. Shafir, H. Soifer, B. D. Bruner, M. Dagan, Y. Mairesse, S. Patchkovskii, M. Y. Ivanov, O. Smirnova, and N. Dudovich, Nature (London) 485, 343 (2012).

[6] K. J. Schafer, J. L. Krause, and K. C. Kulander, in ShortPulse High-Intensity Lasers and Applications, edited by H. A. Baldis (SPIE, Bellingham, WA1993) Vol. 1860, pp. 190-197.

[7] P. B. Corkum, Phys. Rev. Lett. 71, 1994 (1993).

[8] M. Lewenstein, P. Balcou, M. Y. Ivanov, A. L'Huillier, and P. B. Corkum, Phys. Rev. A 49, 2117 (1994).

[9] O. Raz, O. Pedatzur, B. D. Bruner, and N. Dudovich, Nat. Photonics 6, 170 (2012).

[10] B. Shan and Z. Chang, Phys. Rev. A 65, 011804 (2001).

[11] D. B. Milošević and W. Becker, Phys. Rev. A 66, 063417 (2002).

[12] C. Figueira de Morisson Faria, M. Dorr, W. Becker, and W. Sandner, Phys. Rev. A 60, 1377 (1999).

[13] C. Figueira de Morisson Faria, D. B. Milošević, and G. G. Paulus, Phys. Rev. A 61, 063415 (2000).

[14] M. V. Frolov, N. L. Manakov, A. A. Silaev, and N. V. Vvedenskii, Phys. Rev. A 81, 063407 (2010).

[15] M. V. Ammosov, N. B. Delone, and V. P. Krainov, Sov. Phys. JETP64, 1191 (1986).

[16] M. V. Frolov, N. L. Manakov, T. S. Sarantseva, M. Y. Emelin, M. Y. Ryabikin, and A. F. Starace, Phys. Rev. Lett. 102, 243901 (2009).

[17] A. D. Shiner, B. E. Schmidt, C. Trallero-Herrero, H. J. Wörner, S. Patchkovskii, P. B. Corkum, J.-C. Kieffer, F. Légaré, and D. M. Villeneuve, Nat. Phys. 7, 464 (2011).

[18] L. Greenman, P. J. Ho, S. Pabst, E. Kamarchik, D. A. Mazziotti, and R. Santra, Phys. Rev. A 82, 023406 (2010).

[19] S. Pabst, Eur. Phys. J. Spec. Top. 221, 1 (2013).
[20] S. Pabst, L. Greenman, P. J. Ho, D. A. Mazziotti, and R. Santra, Phys. Rev. Lett. 106, 053003 (2011).

[21] S. Pabst, A. Sytcheva, A. Moulet, A. Wirth, E. Goulielmakis, and R. Santra, Phys. Rev. A 86, 063411 (2012).

[22] S. Pabst, L. Greenman, D. A. Mazziotti, and R. Santra, Phys. Rev. A 85, 023411 (2012).

[23] S. Pabst and R. Santra, J. Phys. B 47, 124026 (2014).

[24] S. Pabst, D. Wang, and R. Santra, Phys. Rev. A 92, 053424 (2015).

[25] S. Pabst and R. Santra, Phys. Rev. Lett. 111, 233005 (2013).

[26] P. Salières, A. L'Huillier, and M. Lewenstein, Phys. Rev. Lett. 74, 3776 (1995).

[27] P. Balcou, P. Salières, A. L'Huillier, and M. Lewenstein, Phys. Rev. A 55, 3204 (1997).

[28] C. Jin, H. J. Wörner, V. Tosa, A.-T. Le, J. B. Bertrand, R. R. Lucchese, P. B. Corkum, D. M. Villeneuve, and C. D. Lin, J. Phys. B 44, 095601 (2011).

[29] C. Altucci, R. Bruzzese, C. de Lisio, M. Nisoli, E. Priori, S. Stagira, M. Pascolini, L. Poletto, P. Villoresi, V. Tosa, and K. Midorikawa, Phys. Rev. A 68, 033806 (2003).

[30] V. Tosa, E. Balogh, and K. Kovács, Phys. Rev. A 80, 045801 (2009).

[31] C. Vozzi, M. Negro, F. Calegari, S. Stagira, K. Kovács, and V. Tosa, New J. Phys. 13, 073003 (2011).

[32] P. Balcou, A. S. Dederichs, M. B. Gaarde, and A. L'Huillier, J. Phys. B 32, 2973 (1999).

[33] M. Lewenstein, P. Salières, and A. L'Huillier, Phys. Rev. A 52, 4747 (1995).

[34] U. Andiel, G. D. Tsakiris, E. Cormier, and K. Witte, Europhys. Lett. 47, 42 (1999).

[35] H. Soifer, B. D. Bruner, M. Negro, M. Devetta, D. Faccialà, C. Vozzi, S. de Silvestri, S. Stagira, and N. Dudovich, J. Phys. B 47, 204029 (2014).

[36] C. Vozzi, F. Calegari, E. Benedetti, S. Gasilov, G. Sansone, G. Cerullo, M. Nisoli, S. D. Silvestri, and S. Stagira, Opt. Lett. 32, 2957 (2007).

[37] L. Poletto, G. Naletto, and G. Tondello, Opt. Eng. (Bellingham, Wash.) 40, 178 (2001).

[38] N. Ishii, A. Kosuge, T. Hayashi, T. Kanai, J. Itatani, S. Adachi, and S. Watanabe, Opt. Express 16, 20876 (2008).

[39] Y.-J. Chen, S. Pabst, A. Karamatskou, and R. Santra, Phys. Rev. A 91, 032503 (2015). 